\title{
105 \\ CHANNEL PRE-ALLOCATION FOR SINGLE-HOP WDM NETWORKS WITH MULTIPLE RECEIVERS
}

\author{
Sugang $X U^{1}$ and Yoshiaki TANAKA ${ }^{1,2}$ \\ ${ }^{1}$ Global Information and Telecommunication Institute, Waseda University \\ 1-3-10 Nishi-Waseda, Shinjuku-ku, Tokyo, 169-0051 Japan \\ ${ }^{2}$ Advanced Research Institute for Science and Engineering, Waseda University \\ 17 Kikuicho, Shinjuku-ku, Tokyo, 162-0044 Japan \\ Te1/Fax: +81-3-5286-8389, e-mail: xsg@aoni.waseda.jp, ytanaka@waseda.jp
}

\begin{abstract}
WDM optical networks represent the direction to the future high capacity access network applications. In this paper we investigate the influence of tuning latency on the performance of different architectures of singlehop WDM star networks. Two configurations are mainly considered: one slowly tunable transmitter and multiple fixed receivers (STT/MFRs) and single tunable transmitter and single fixed receiver (TT/FR). For both configurations one home channel is pre-allocated to each node. Assuming the traffic is non-uniformly distributed, an optimal channel allocation with a simple Mixed Integer Linear Programming model is proposed for STT/MFRs. To investigate the performance, evaluation through simulations is done in terms of network throughput, packet delay. The results show us $T T / F R$ is tuning latency sensitive, while STT/FR is not a tuning latency sensitive architecture. TT/FR configuration requires very quidkly tunable transmitters, while STT/MFRs configuration can make use of the off-theshelf low cost slowly tunable optical components to yield good performance.
\end{abstract}

\section{INTRODUCTION}

WDM optical networks represent the direction to the future high capacity access network applications. For local access network, the star-coupled single-hop configuration was shown to exhibit superior fanout characteristics over optical bus-based systems [1][2]. Media access control protocols developed for photonic star-coupled WDM networks may be generally classified into two types strategies: reservation and preallocation strategies [2]-[4]. Reservation techniques may use one channel (control channel) to reserve remaining channels (data channels) for data packet transmission [5]-[7]. Media access control protocols are required to provide arbitration on both the data and control channels. Pre-allocation strategies pre-allocate the channels to the nodes, each node has one channel as home channel that it uses for data transmissions. Reservation protocols are often more complex

0-7803-8560-8/04/\$20.00@ (c) 2004IEEE than pre-allocation protocols. While pre-allocation approaches are very promising due to their low implementation and operation complexity.

In this paper we investigate the pre-allocation strategies in a star-coupled single-hop WDM network, especially for an architecture in which each node has one slowly tunable transmitter and multiple fixed receivers (STT/MFRs). One home channel is pre-allocated to each node, and transmitter of each node will be tuned to that channel for packet transmission. Differ from the past works shown above that the tuning latency is not mainly considered (using the very quickly tunable transmitter/receiver is assumed), we assume that the tuning latency is not neglectable. One objective is to observe the influence of tuning latency on the performance of different architectures, and select a tuning latency insensitive architecture. Long tuning latency would cause poor performance when tuning packet by packet is necessary. The motivation of employing STT/MFRs is to make use of the off-the-shelf low cost slowly tunable transmitter and multiple low cost fixed receivers to create high performance systems and reduce the control complexity, when the very quickly tunable transmitters and/or receivers are still expensive. We assume that traffic is non-uniformly distributed, there exists certain traffic pattern for certain period of time, and this pattern would change from time to time. With this non-uniformly distributed traffic, we proposed an optimal channel allocation with a simple Mixed Integer Linear Programming (MILP) model based on STT/MFRs configuration. The rest of paper is organized as follows: Section 2 provides information on optical components and the network architecture. Section 3 describes the optimal pre-allocation approach for slotted-ALOHA protocol in MILP model. Section 4 provides the performance evaluations of network in terms of the network throughput, packet delay through simulation studies. Section 5 summarizes the paper.

\section{NETWOKR ARCHITECTURE WITH LOWER CONTROL COMPLEXITY}

Generally, there are several configurations of star coupled single-hop WDM network. For example sin- 
gle tunable transmitter/single fixed receiver (TT/FR), or single fixed transmitter/single tunable receiver (FT/TR), or single tunable transmitter/single tunable receiver (TT/TR), or single fixed transmitter/Multiple fixed receiver (FT/MFRs). For the savings of the cost, most studies are focused on TT/FR or FT/TR configurations. Although the amount of key components (transmitter/receiver) is small, these configurations require very quickly tunable transmitters or receivers. With FT/TR configuration, generally, an extra control channel is used for packet transmission control, reservation is necessary before packet transmission, it introduces control complexity. For FT/MFRs configuration, it can not be adapted to traffic changes, the resource utilization could not be optimized.

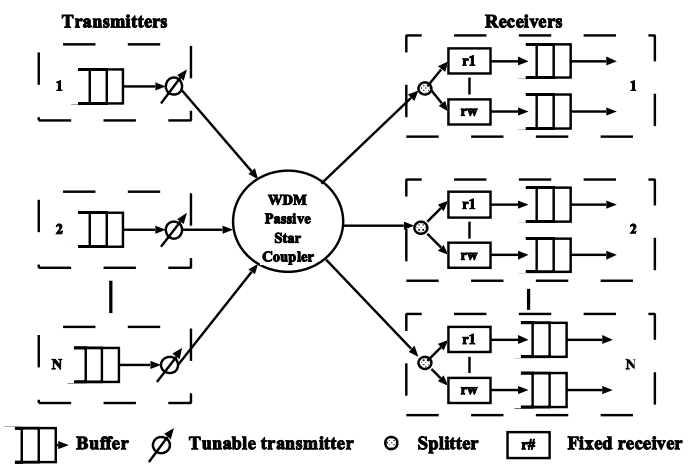

Fig. 1. Network architecture.

In this paper, we consider a packet switched singlehop WDM star network with $N$ nodes, in which each node has one slowly tunable transmitter and multiple fixed receivers (STT/MFRs), please see Fig.1. Network supports $W<N$ wavelength channels, all channels are used for data transmission, no control channel is required, it reduces the control complexity. One channel is pre-allocated to each node, and transmitter of each node will be tuned to that channel for packet transmission. The transmitters are tunable over the entire range of wavelengths, and the transmitters are slowly tunable. Let $\Delta_{t}$ denote the normalized tuning latency of transmitter, expressed in units of packet transmission time (slot). We assume that $\Delta_{t} \geq 0$, where $\Delta_{t}$ is a small integer. A special case that $\Delta_{t}=0$ means an ideal transmitter without tuning latency that can tuning to target wavelength immediately. The motivation of employing slowly tunable transmitters is the significant savings in the cost.

At each node, packets will be stored into a buffer (FIFO) before transmission. One packet will be transmitted in one time slot. Each node is of $W$ fixed tuned receivers. All receivers can be used to receive packets simultaneously from each channel. It removes receiver contention and requirement of receiver tuning that occurs in single tunable receiver configuration. Also, each node can detect whether outgoing packet is collided with other packets easily by monitoring its home channel after packet sending, while in the single re-
106

ceiver configuration it would be more complicated to verify whether the outgoing packet is collided. So performance will be improved significantly. Also multicast support would be required for efficient resource utilization, with multiple fixed tuned receivers configuration the multicast can be implemented easily, and the control complexity would be reduced significantly. Although employing multiple fixed receivers will introduce extra costs into systems comparing to single receiver system, it is believed that the cost of fixed receivers (especially for the low speed fixed receiver) and system integration is turning down. Considering the contributions on lower control complexity and higher performance, based on the performance-cost considerations, it is possible to use multiple fixed receivers system with lower cost in the near future. We try to make use of the off-the-shelf low cost slowly tunable transmitter and multiple low cost fixed receivers instead of the very expensive quickly tunable transmitter and/or receiver. In fact TT/FR configuration yields good performance only when the very quickly tunable transmitter is employed, the performance evaluations are shown in section 4 .

Assuming the traffic is non-uniformly distributed, there exist certain traffic patterns for certain periods of time, expressed by an $N \times N$ traffic demand matrix $\Lambda=\left[t_{i j}\right]$, where $t_{i j}$ denotes the average traffic originating from node $i$ and terminating at node $j$. This pattern would change from time to time. For example users are downloading big files or watching online TV or on-demand video contents, the continuous heavy traffic from several nodes (attached with contents servers, cache servers, back-bone Gateway, etc.) will keep for several time periods. Because each node has $W$ fixed receivers, all packets that are successfully transmitted on each channel would be monitored by each node. The traffic information can be gathered at each node. Centralized traffic measurement at an extra control node is not necessary. With distributed traffic measurement at each node the system complexity would be further reduced. Each node maintains the same traffic matrix $A$ all the time. This information can be used to optimize the channel allocation, which will be talked about in the next section.

\section{CHANNEL PRE-ALLOCATION}

For the packet transmission, slotted-ALOHA protocol is used. At the beginning of each slot, node $i$ with packets in buffer to be sent will transmit the packet on its home channel. Channel collisions occur when two or more nodes attempt transmission on the same channel at the same time. There is no receiver contention due to $W$ fixed receivers are employed. A successful transmission takes place when a single packet is transmitted on a channel. Assuming the traffic is non-uniformly distributed, there exists certain traffic pattern for certain periods of time. The home channel allocation is computed periodically if the traffic pattern changes are detected. For STT/MFRs configuration we propose an optimal channel allocation with 
a simple Mixed Integer Linear Programming (MILP) model. The objective is to achieve the load balance among all channels shown as Eq.(1), constraints are shown as Eq.(2).

Objective:

$$
\operatorname{minimize}: \max _{i}\left(\sum_{j=0}^{N-1} c_{i}^{j} t_{j}\right) \quad(0 \leq i \leq W-1)
$$

Subject to:

$$
\sum_{i=0}^{W-1} c_{i}^{j}=1 \quad(0 \leq j \leq N-1)
$$

where indicator $c_{i}^{j}\left(c_{i}^{j}=0\right.$ or 1$)$ denotes the channel allocation, $c_{i}^{j}=1$ if channel $i(0 \leq i \leq W-1)$ is assigned to node $j(0 \leq j \leq N-1)$, otherwise $c_{i}=0$; $t_{j}$ denotes the sum of traffics originating from node $j$, $t_{j}=\sum_{i} t_{j i}(0 \leq i \leq N-1,0 \leq j \leq N-1)$. Constraints in Eq.(2) express that only one channel will be assigned to each node. The home channel is fixed. Due to the $W$ fixed receivers are employed the MILP optimization model complexity is drastically reduced. Amount of both variables and constraints are reduced significantly. With a network of 100 nodes (evaluated in section 4), the optimal solution can be found within minutes on a Pentium-4 (1.8G) based Linux PC.

During a certain range of time without obvious traffic pattern changes detected, the optimal channel preallocation is computed and fixed. When obvious traffic pattern changes are detected by each node, the channel allocation is recomputed locally at each node to achieve optimal resource utilization dynamically. With the same input traffic pattern and the same algorithm, the optimal solutions yielded from each node are consistent. Because that the channel allocations are fixed within certain time period, tuning from one channel to another is not necessary. Retuning only occurs after channel allocation re-computation, say the traffic pattern changes. For the space limitation, the retuning optimization problem that minimizes the retuning requirement and further fast algorithm are out of the scope of this paper.

In this paper, we compare performance between pre-allocation for STT/MFRs configuration and interleaved pre-allocation for TT/FR configuration. Because single fixed receiver is used at each node, the home channel is fixed and assigned by Eq.(3), where $c_{t}$ denotes the channel allocation for node $i$. The system cannot be optimized corresponding to traffic pattern changes. Note that each destination node would have different home channel to receive packets, node with packets to send has to tune its transmitter to destination node home channel before packets transmissions. Because packets in buffer generally are not sorted by each destination, tuning packet by packet at each transmitter would be required.

$$
c_{i}=i \bmod W
$$

\section{PERFORMANCE EVALUATION}

In this section, the performance evaluations of preallocation for STT/MFRs and TT/FR configuration through simulations are provided. The performance metrics are network throughput and average packet delay. Throughput is defined as average successfully transmitted packets per unit time slot. Average packet delay is defined as average amount of time (counted in unit time slot) spent in transmitter queue and transmission time (one slot) for each successfully transmitted packet. Due to that the propagation delay along each fiber is fixed, it is not counted here. Buffer size is limited with maximum 1000 packets. Packet size is assumed to be 1500 Bytes. The transmission speed is assumed to be $10 \mathrm{Gbps}$. Each time slot is about $1.2 \mu \mathrm{s}$. Tuning latency $\Delta_{t}$ of transmitter will be set from 0 to 4 within $10 \mu$ s order tuning latency, say from the ideal transmitter without tuning latency to the slowly tunable transmitter with longer tuning latency. Network is of 100 nodes $(N=100) .10$ and 50 wavelengths are used for observations $(W=10,50)$. At the beginning, non-uniform traffic demand matrix $\Lambda$ is generated. Interval of packet transmission requests arrival at each node is exponentially distributed. Through simulations four configurations are compared: pre-allocation for STT/MFRs is denoted by STT/MFRs; interleaved preallocation for TT/FR configuration with tuning latency $\Delta_{t}=0$ is denoted by TT/FR( 0$)$; TT/FR configuration with tuning latency $\Delta_{t}=2$ is denoted by TT/FR(2); TT/FR configuration with tuning latency $\Delta_{t}=4$ is denoted by TT/FR(4).

Figure. 2and 3 illustrate the network throughput vs. network load (total load of $N$ nodes, where load is defined as average packet transmission requests arriving within one slot) comparisons between STT/MFRs and TT/FR configurations with different tuning latency for $W=10$ and 50 cases, respectively. Optimal preallocation for STT/MFRs configuration outperforms the interleaved pre-allocations for TT/FR configuration, one reason is that the loads among each channel are well balanced, another reason is the multiple receivers receive packets simultaneously from each channel, while for the TT/FR configuration, one node just can receive one packet at each slot. For STT/MFRs configuration, without tuning requirement in certain time period, transmitter tuning latency has no relation to performance. On the contrast, for TT/FR configuration almost packet by packet tuning is required, with longer tuning latency (for example $\Delta_{t}=4$ comparing to $\Delta_{t}=0$ ) transmitter has to spend more time on tuning, the packet has to stay in queue longer waiting for transmission, the throughput is lower.

Figure.4 illustrates the comparisons of throughput vs. average packet delay. Without tuning requirement optimal pre-allocation for STT/MFRs keeps small delay when throughput is increasing. While with longer tuning latency the interleaved pre-allocation for TT/FR will cause quite long delay, more packets are waiting in queue for tuning. The buffer is easy to be overflowed. Throughput is limited. 


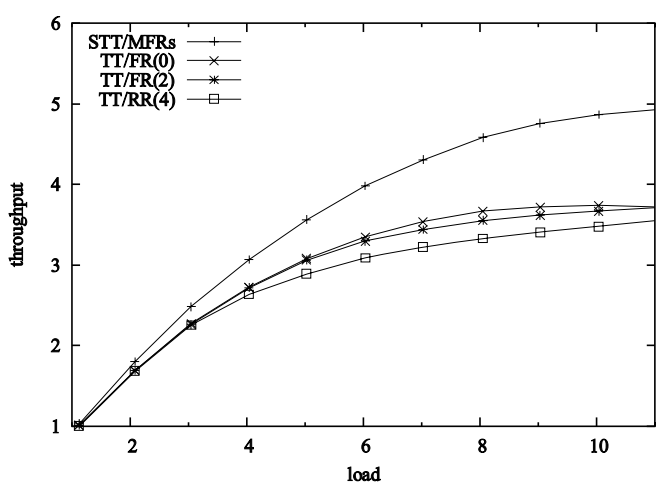

Fig. 2. Throughput comparisons for $W=10$ case.

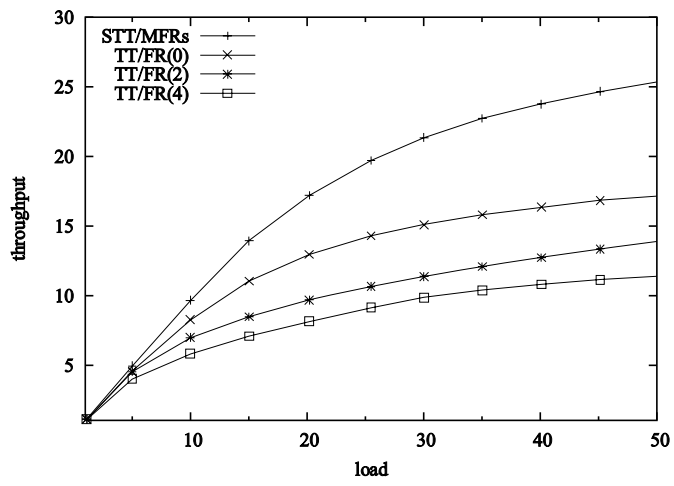

Fig. 3. Throughput comparisons for $W=50$ case.

Also from Fig. 4 we can find with more channels (for example $W=50$ comparing to $W=10$ ) performance of optimal pre-allocation for STT/MFRs can be improved significantly. On the other hand for the TT/FR configuration, only the ideal transmitter without (or very small) tuning latency could make use of more channels to improve performance. With longer tuning latency (for example $\Delta_{t}=4$ ) the performances of both $W=10$ and $W=50$ cases are almost the same. It shows us that with long tuning latency, more channels could not be used efficiently. For TT/FR configuration to yield good performance and efficient wavelength utilization quickly tunable transmitter is necessary. If the quickly tunable transmitter is not available, the tuning latency has to be considered, STT/MFRs with optimal pre-allocation would be a good choice.

\section{CONCLUSIONS}

In this paper we investigated a single-hop WDM star network with a passive star-coupled WDM based configuration, in which each node has one slowly tunable transmitter and multiple fixed receivers. One home channel is pre-allocated to each node. Assuming the traffic is non-uniformly distributed, an optimal channel allocation with a simple MILP model is proposed. With STT/MFRs configuration and pre-allocation dis-
108

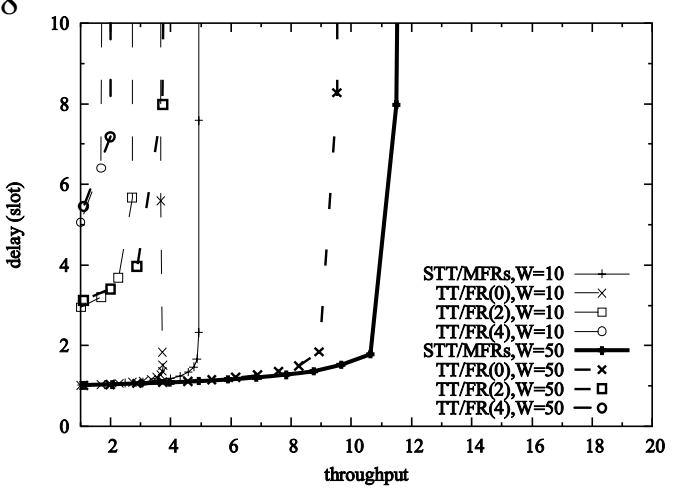

Fig. 4. Throughput vs. delay comparisons.

tributed control, extra central control node is not required, reliable and high performance system with low cost can be achieved. For TT/FR configuration to yield good performance and efficient wavelength utilization very quickly tunable transmitter is necessary.

\section{ACKNOWLEDGEMENTS}

The authors would like to thank Mr. Ohgiya for his help to calculate the performance.

\section{REFERENCES}

[1] M. M. Nassehi, F. A. Tobagi, and M.E. Marhic, "Fiber optic confi gurations for local area networks," IEEE J. Select. Areas Commun., vol. SAC-3, pp.941-949, November 1985.

[2] P. W. Dowd, "Optical bus and star-coupled parallel inter-connection," 4th International Parallel Processing Symposium, pp.824-838, April 1990.

[3] P. W. Dowd, "Random access protocols for high speed interprocessor communication based on a passive star topology," IEEE J. Lightwave Tech., vol. 9, pp.799808, June 1991.

[4] P. W. Dowd, "High performance interprocessor communication through optical wavelength division multiple access channels," 18th International Symposium on Computer Architecture, pp.96-105, May 1991.

[5] I. M. I. Habbab, M. Kavehrad, and C. E. W. Sundberg, "Protocols for very high-speed optical fi ber local area networks using a passive star topology," IEEE J. Lightwave Tech., vol. LT-5, pp. 1782-1793, December 1987.

[6] M. S. Chen, N. R. Dono, and R. Ramaswami, "A media access control protocol for packet switched wavelength division multiaccess metropolitan area network," IEEE J. Select. Areas Commun., vol. 8, pp. 1048-1057, August. 1990.

[7] G. Sudhakar, N. Georganas, and M. Kavehrad, "Slotted Aloha and Reservation Aloha protocols for very high-speed optical fi ber local area networks using passive star topology," IEEE J. Lightwave Tech., vol. 9, pp.1411-1422, October 1991. 